\title{
Similarity of the Jovian satellite footprints: Spots multiplicity and dynamics
}

\author{
B. Bonfond ${ }^{a, *}$, D. Grodent ${ }^{a}$, S.V. Badman ${ }^{b}$, J. Saur ${ }^{c}$, J.-C. Gérard ${ }^{a}$, A. Radioti ${ }^{a}$ \\ a Space sciences, Technologies and Astrophysics Research (STAR) Institute, LPAP, Université de Liège, Liège, Belgium \\ ${ }^{\mathrm{b}}$ Department of Physics, Lancaster University, United Kingdom \\ ' Institut für Geophysik und Meteorologie, Universität zu Köln, Cologne, Germany
}

\section{A R T I C L E I N F O}

\section{Article history:}

Received 2 August 2016

Revised 2 December 2016

Accepted 5 January 2017

Available online 10 January 2017

\section{Keywords:}

Jupiter

Aurora

Footprint

Io

Europa

Ganymede

\begin{abstract}
A B S T R A C T
In the magnetospheres of Jupiter and Saturn, the intense interaction of the satellites Io, Europa, Ganymede and Enceladus with their surrounding plasma environment leaves a signature in the aurora of the planet. Called satellite footprints, these auroral features appear either as a single spot (Europa and Enceladus) or as multiple spots (Io and Ganymede). Moreover, they can be followed by extended trailing tails in the case of Io and Europa, while no tail has been reported for Ganymede and Enceladus, yet. Here we show that all Jovian footprints can be made of several spots. Furthermore, the footprints all experience brightness variations on timescale of 2-3 $\mathrm{min}$. We also demonstrate that the satellite location relative to the plasma sheet is not the only driver for the footprint brightness, but that the plasma environment and the magnetic field strength also play a role. These new findings demonstrate that the Europa and Ganymede footprints are very similar to the Io footprint. As a consequence, the processes expected to take place at Io, such as the bi-directional electron acceleration by Alfvén waves or the partial reflection of these waves on plasma density gradients, can most likely be extended to the other footprints, suggesting that they are indeed universal processes.
\end{abstract}

(c) 2017 Elsevier Inc. All rights reserved.

\section{Introduction}

A satellite footprint is an auroral emission related to the electromagnetic interaction between a moon and the magnetospheric plasma that surrounds the planet. These emissions appear close and downstream (as explained below) of both ionospheric ends of the magnetic field lines connecting the moon to the planet. The first one to be discovered was the Io footprint (IFP), initially detected in the infrared domain at $3.4 \mu \mathrm{m}$ (Connerney et al., 1993), then in the Far-UV (FUV; 120-170 nm) (Prangé et al., 1996; Clarke et al., 1996) and in the visible domains (Vasavada et al., 1999). The Europa and Ganymede footprints have later been detected simultaneously with the Hubble Space Telescope (HST) (Clarke et al., 2002). The discovery of the Enceladus footprint on Saturn required close-up observations from the UVIS instrument on board Cassini (Pryor et al., 2011).

The Io footprint is made of at least three distinct spots and an extended tail (Gérard et al., 2006; Bonfond et al., 2008). A shorter tail has also been seen for the Europa footprint (EFP) (Grodent et al., 2006) and a secondary spot has also been identified in the

\footnotetext{
* Corresponding author.

E-mail address: b.bonfond@ulg.ac.be (B. Bonfond).
}

southern hemisphere for the Ganymede footprint (GFP) (Bonfond et al., 2013a).

Being the brightest of all footprints, the Io footprint has been studied with the most details. According to the scenario depicted by Bonfond et al. (2013b) and Hess et al. (2013a), the multiple spots of the IFP arise from a chain of processes. The foundations of this scenario rest on two categories of arguments: one based on geometry, which involves the location and relative motion of the footprint's features (e.g. Connerney and Satoh, 2000; Gérard et al., 2006; Bonfond et al., 2008), and one based on the physical processes at play in the satellite-magnetosphere interaction (e.g. Goldreich and Lynden-Bell, 1969; Neubauer, 1980; Hess et al., 2010), which controls the brightness variations of these auroral features.

The magnetic moment of Jupiter is inclined by about $9^{\circ}$ relative to its rotation axis. As a consequence, the magnetospheric plasma is densest along the centrifugal equator, which is tilted compared to the spin equator. The mass loss of Io forms a plasma torus at Io's L-shell, which transforms into a plasma sheet at larger distances. At the radial distance of Io, the magnetospheric plasma almost corotates with the planet. Since Io rotates more slowly around the planet compared to the magnetospheric plasma, it forms an obstacle to the plasma flow, which is slowed down and diverted 
around the moon (Saur et al., 2004; Jia et al., 2009, and references therein). This interaction generates a disturbance propagating along the magnetic field lines in the rest frame of the moving plasma in the form of Alfvén waves (Neubauer, 1980). Alfvén waves propagate at the Alfvén velocity, which is proportional to the magnetic field strength and inversely proportional to the square of the mass density. The ratio of plasma velocity relative to the satellite $v_{0}$ to Alfvén velocity $v_{A}$ is the Alfvén Mach number $M_{A}=\frac{v_{0}}{v_{A}}$. The loci of the perturbations, called Alfvén wings, are inclined in the downstream direction compared to the undisturbed magnetic field lines by the angle $\Theta_{A}=\arctan M_{A}$. As the Alfvén waves leave Io, their large scale components evolve into smaller scale filamented Alfvén waves (Chust et al., 2005). They then reach density gradients at the boundaries of the plasma torus and these waves get partially reflected back into the torus (Neubauer, 1980). When the remaining transmitted Alfvén waves reach the high latitude region, approximately $1 R_{J}$ (Jovian radius) above the surface, they enter a region where wave particle interactions accelerate electrons in both directions (Jones and Su, 2008; Hess et al., 2010). A fraction of these electrons directly precipitates into the closest auroral region and form the Main Alfvén Wing spot (MAW spot). The electrons accelerated in the opposite direction form an electron beam which follows the magnetic field line from the acceleration region above the planet surface in one hemisphere to the opposite hemisphere. A fraction of these electrons are mirrored back, forming a partially trapped bi-directional population downstream of Io (Williams et al., 1996; Jacobsen et al., 2010). Another fraction precipitates in the hemisphere opposite from the initial acceleration region, forming a Trans-hemispheric Electron Beam spot (TEB spot) (Bonfond et al., 2008). The Alfvén waves that have been partially reflected on one torus boundary may still get partially transmitted at the opposite boundary and end up forming the reflected Alfvén wing spot (RAW spot). The electron beams approximately follow the magnetic field lines because of the large kinetic velocities of these electrons. On the other hand, the Alfvén wings are inclined with respect to the magnetic field because of the slower Alfvén velocity in the torus. These effects contributes to the complexity of the footprint patterns. Finally, in addition to the Alfvénic acceleration which provides most of the energy to the precipitating electrons, transient and upward migrating double layers structures observed from 0.07 to $0.3 R_{J}$ above the planet may provide some additional energy to these electrons (Hess et al., 2009). The appearance and disappearance of these structures could be the source of the variability of the spots' brightness on timescale of minutes (Bonfond et al., 2007, 2013b).

Because of the tilt of the Jovian magnetic field relative to the orbital plane of the satellites, the latitude of the moons relative to the centrifugal equator varies approximately sinusoidally with their System III $\left(S_{I I I}\right)$ longitude. The moons thus encounter plasma density variations as they move up and down the plasma torus/sheet. These variations impact both the strength of the electromagnetic interaction at the moon, and the path of the Alfvén wings. Ultimately they control the relative motion of the MAW, TEB and RAW spots (see Fig. 1 and the animations provided as auxiliary material in Bonfond et al., 2013a). Moreover, the absolute spots' brightness varies with the $S_{I I I}$ longitude of Io and Ganymede (Bonfond et al., 2013b; Grodent et al., 2009). In the case of Io, the relative brightness of the spots varies with $S_{I I I}$ as well. However, the location of Io relative to the center of the plasma torus is not the only parameter controlling the brightness of the spots. For example, the brightness of the Io footprint MAW southern spot is much higher around $110^{\circ} S_{I I I}$ than around $290^{\circ} S_{I I I}$, while Io is right in the center of the torus in both cases. First, the local interaction is controlled by the plasma density, the magnetic field strength, and the ionosphere of Io. All these parameters vary with $S_{I I I}$ and which partially explains the difference between the two longitude ranges
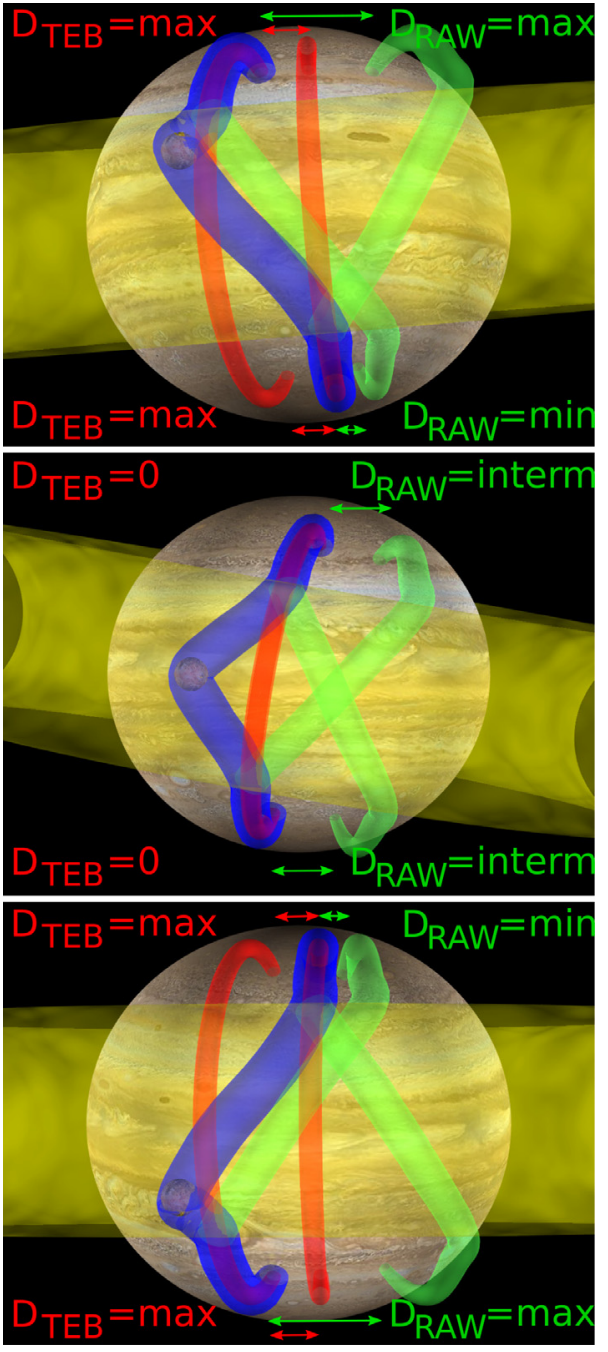

Fig. 1. Scheme of the Alfvén wing reflection pattern and Trans-hemispheric Electron Beams (TEB, shown in red) when Io is in its northern-most (top), central (middle) or southern-most position relative to the plasma torus (shown in yellow). The Main Alfvén Wing (MAW) is shown in blue and the reflected one is shown in blue. On the top panel, in the North, the distance between the MAW spot and the TEB spot $\left(D_{T E B}\right)$ is maximal as well as the distance between the MAW spot and the RAW spot $\left(D_{R A W}\right)$. Note that in a linear case, $\max D_{R A W} \sim 2 \max D_{\text {TEB }}$. In the South, $D_{\text {TEB }}$ is also maximal, but the TEB spot is now upstream of the MAW spot. $D_{R A W}$ reaches its minimum. When Io is in the center of the torus (middle panel) the situation in the two hemispheres is symmetric, and the TEB spots are merged with the RAW spots. (For interpretation of the references to color in this figure legend, the reader is referred to the web version of this article.)

(Saur et al., 2013). However, alone, these variations of the local interaction are not sufficient to account for the observations and couldn't explain the observed North/South brightness asymmetries (Bonfond et al., 2013b). Indeed, other important processes (waves transmission, electron acceleration, mirroring, etc.) occur further along the wings which are controlled by the magnetic field as well as the plasma densities and temperatures, e.g. in the torus and in the acceleration region. The latter appears to significantly contribute to the variability (Hess et al., 2010; 2013a). This indicates that it is not only the strength of the interaction at the satellite that controls the spots' brightness but that the longitudinal and North-South asymmetries of the magnetic field also play a major role.

In addition to these already complex variations as a function of the longitude, the brightness of the Io footprint spots may also display changes from one observation to another due to stochastic variability in Jupiter's magnetosphere. This was illustrated by 
Bonfond et al. (2012), as the Io footprint crossed the path of an emission blob most probably related to an injection signature (Mauk et al., 2002; Dumont et al., 2014). On this set of FUV images, the IFP nearly disappears as it crosses the blob before re-emerging outside of it. The model of Payan et al. (2014) explains this behavior through a localized increase of the torus density resulting in a stronger trapping of the Alfvén waves within the torus. Another study by Hess et al. (2013b) also agrees that an increased torus density would decrease the wave transmission across the torus boundary. However these authors favor a decrease of the inertial Alfvén waves' electron acceleration process accompanying an enhanced high energy electron density at high latitude during injections as the most likely explanation for the observed disappearance of the IFP.

If this scenario involving a chain of processes is correct for Io, then it should also apply for Europa and Ganymede. In the present study, we present new observations of the complex morphology and brightness evolution of the Europa and Ganymede footprints. These observations show that most phenomena described above concerning the Io footprint are indeed also observed for Europa and Ganymede. They thus indicate that the processes at play at Io are also valid for the other Jovian satellites and thus are most likely universal. Furthermore, Alfvén wave reflections on the density gradients and the bi-directional electron acceleration by dispersive Alfvén waves would also explain the observed behavior of the Europa and Ganymede footprints.

\section{Image processing}

Most of the results presented here arise from two recent Hubble Space Telescope (HST) campaigns (GO 12883 and GO 13035) dedicated to the observation of the FUV aurorae at Jupiter. Since HST is an orbiting telescope, the observing blocks attributed to each campaign correspond to an orbit. In the present study, we focus on the imaging observations, which have been carried on with the time-tag mode and the Strontium Fluoride filter $(\sim 130$ $182.5 \mathrm{~nm}$ ) of the Space Telescope Imaging Spectrograph (STIS) FUV-MAMA (Multi-Anode Microchannel Array) channel. This mode allows 1) a high spatial resolution with a platescale of 0.024 arcs per pixel and point spread function of approximately the same size at full width at half maximum (FWHM), 2) a time resolution up to $10 \mathrm{~s}$ for images with a fair signal to noise ratio and 3) up to 45 min long continuous sequences (Bonfond et al., 2011). In practice, in order to meet their various science objectives, most observations campaigns were not designed in such a way that HST would continuously stare at one hemisphere for such a long time. The sequences considered here are between 6.5 and 24.3 minutelong. The GO 12883 campaign was dedicated to alternating sequences from the southern and northern hemisphere. During campaign GO 13035, spectral observations have been inserted between two imaging sequences of the northern hemisphere (Badman et al., 2016; Tao et al., 2016). For parts of our study, we also include similar observations from the HST GO 7308, GO 8171, GO 8657 and GO 9685 programs to improve our statistics. Some of these images were acquired as standard "ACCUM" images rather than time-tag sequences. A sub-set of those were acquired with the CLEAR filter ( $\sim 115-185 \mathrm{~nm})$, which has a slightly wider bandwidth including the $\mathrm{H}$ Lyman- $\alpha$ line.

The dark counts, flat field and geometry corrections are applied to every 10-s long sub-exposures extracted from the timetag sequences using the typical "Calstis" procedure from the Space Telescope Science Institute. Conversion coefficients from counts on the detector to the emitted brightness and power in the whole $\mathrm{H}_{2} \mathrm{Ex}$ treme and FUV range account for the synthetic $\mathrm{H}_{2}$ spectrum as in Gustin et al. (2012). The color ratio of the EFP has not been measured yet and the color ratio of the GFP appears to be variable, since it has previously been measured to be as low as 1.8 (Gérard et al., 2014) and as high as 6.7 (Gustin et al., 2016) on different datasets using the same technique. For this reason, we chose here to use a canonical value of 2.5 . It should however be noted that changing the color ratio would only modify the observed brightness and emitted power reported here by $15 \%$, and it would not impact our conclusions.

In light of the findings based on this new dataset, it is sometimes found useful to complement the analysis with HST FUV images acquired with the ACS (Advanced Camera for Surveys) instrument. The images, acquired in "ACCUM" mode with either the F115LP ( $\sim 115-200 \mathrm{~nm})$ or the F125LP $(\sim 125-200 \mathrm{~nm})$ filters, are also processed using the pipeline from the Space Telescope Science Institute before converting counts to brightness or power units using the appropriate coefficients from Gustin et al. (2012).

Because the planetary limb is less crisp on STIS images than on ACS images, the determination of the planetary center on the images (necessary for locating the auroral features on the planet) using an automated limb fitting method (Bonfond, 2009) is sometimes found too inaccurate and the fit has to be refined manually. The auroral emissions are isolated from the planetary background using the method described in Bonfond et al. (2011).

\section{The satellite footprint brightness}

\subsection{Sudden dimming of the satellite footprint power}

The power emitted by the satellite footprint spots in each hemisphere essentially varies with the $S_{I I I}$ longitude of the satellite. Furthermore, sharp variations of the spots' brightness have been observed on timescales of minutes or tens of minutes (Bonfond et al., 2007, 2013b; Grodent et al., 2009). In the case of the Ganymede footprint, Grodent et al. (2009) suggested that brightness fluctuations within $10-40 \mathrm{~min}$ are related to the encounter between Ganymede and plasma injections. Furthermore, Bonfond et al. (2012) reported a case of disappearance of the Io footprint as it was crossing a blob of auroral emission located at an unusually low latitude.

Fig. 2 shows an example of temporary disappearance of the Ganymede footprint's spot that took place on 11 January 2014. This series of 3 images comes from 2 consecutive timetag sequences from the same HST orbit. Yellow arrows indicate the Ganymede footprint main spot as it crosses a faint and $S_{I I I}$ fixed blob of emission in the outer emissions region. In the first image, which corresponds to the first $100 \mathrm{~s}$ of the first sequence (at 19:39UT), the total power of the GFP main spot is $\sim 7.0 \mathrm{GW}$ and its apparent peak brightness is $1100 \mathrm{kR}$. The maximum brightness in the blob region is $360 \mathrm{kR}$. The second image corresponds to the first $100 \mathrm{sec}$ of the second time-tag sequence and is taken at 20:09 (i.e. $30 \mathrm{~min}$ after the first image). In this image, the GFP spot is now located in the middle of the blob, and the peak brightness of the merging of the two features is $540 \mathrm{kR}$ only (i.e. much less than the sum of both GFP and blob brightness and even much less than the initial GFP brightness). The decrease of the GFP spot's brightness is already apparent at the end of the first sequences, starting around 19:48 as it enters the blob. When it re-emerges from the other side of the blob the peak brightness suddenly increases (around 20:13) and the spot head is then followed by a faint tail connected to the blob. The third image corresponds to the last 100s of the second time-tag sequence and the integrated power over then spot is at least $5.2 \mathrm{GW}$ and the peak brightness is $1470 \mathrm{kR}$.

This case of footprint extinction, lasting for $\sim 25 \mathrm{~min}$ is very similar to the one already observed for the IFP and the process at play is most likely the same. Additionally, these clear cases of relationship between the footprints' brightness and the local plasma environment may explain the large scatter of brightness for 

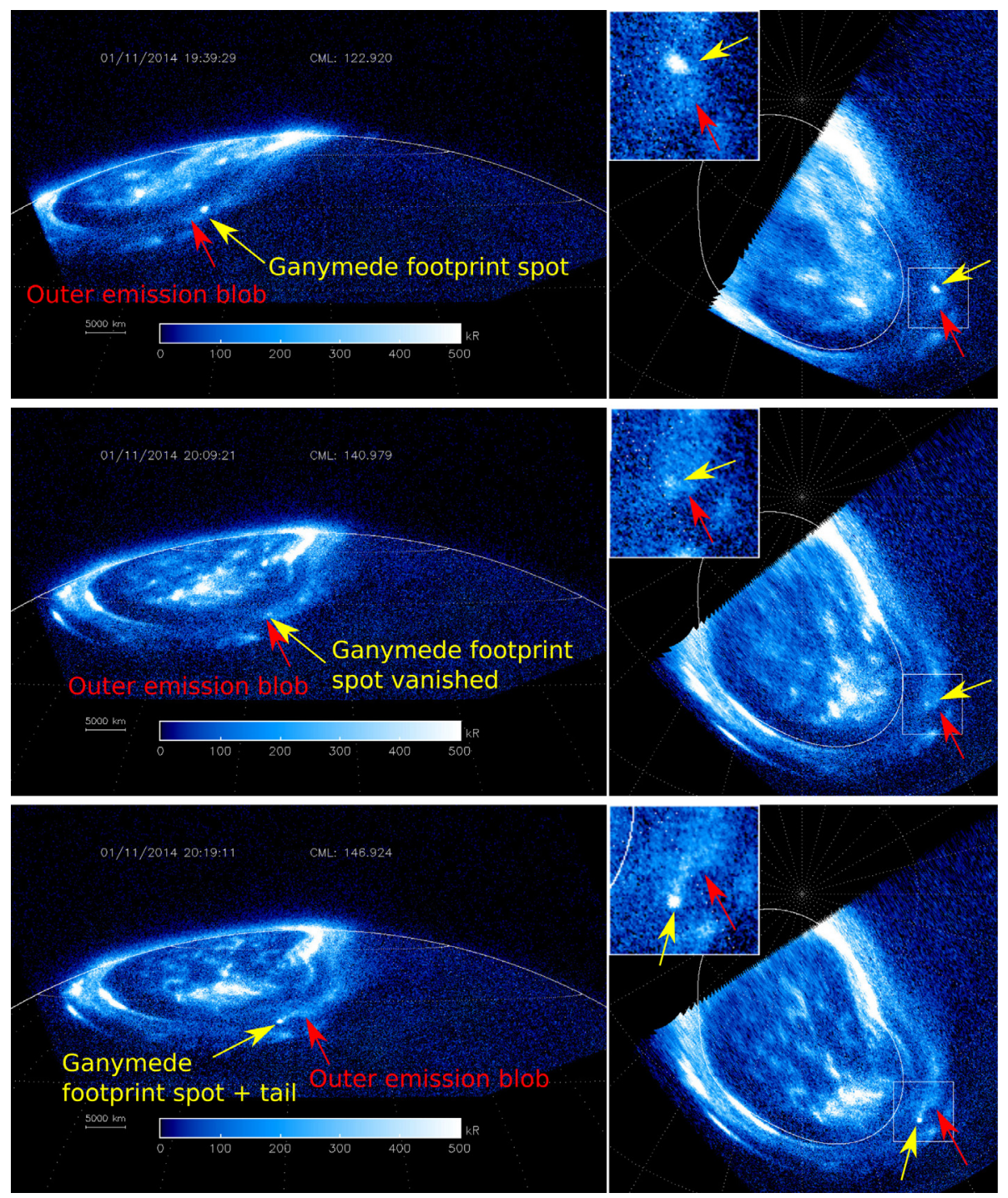

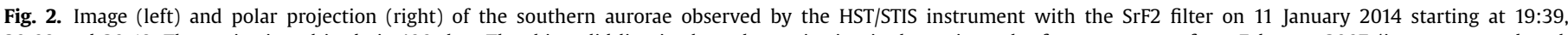

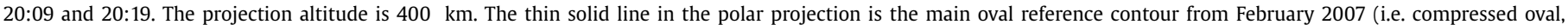
Bonfond et al., 2012). In the middle of the HST orbit, the Ganymede footprint vanishes as it crosses a faint outer emission blob.

the Europa and Ganymede footprints compared to the Io footprint (Grodent et al., 2009; Wannawichian et al., 2010). Indeed, the injection signatures are very frequent and located poleward of the Io footprint contour up to the main emission (Dumont et al., 2014). It should be noted that the case of a blob located as equatorward as the Io footprint (i.e. as radially inward as Io in the equatorial plane) as described in Bonfond et al. (2012) is unique in the whole HST FUV image database.

\subsection{North-South comparisons of the GFP and EFP emitted power}

Visits from the GO 12883 HST campaign alternated images of the southern $(\mathrm{S})$ and northern $(\mathrm{N})$ hemispheres five times in a S$\mathrm{N}-\mathrm{S}$ sequences and then twice in a S-N sequence. Ten minutes separate the end of a timetag sequence and the beginning of the next one, thus allowing a direct comparison of the emitted power of the Ganymede footprint between the hemispheres. The footprint spots are selected manually on summed images after compensating for the motion of the footprint from frame to frame. In order to account for the background contamination, we manually selected an area of the same shape and size just next to the spot and removed the power from this area from the one of the spot in con- sideration. On two cases, it was also possible to apply the same method for the EFP spot. The results for the EFP main spots are shown on Fig. 3. On this figure, it can be seen that, for a given orbit, the northern spot was most of the time dimmer than the southern one in the longitude range under consideration. Around $110^{\circ} S_{I I I}$, the satellite exits the center of the plasma sheet on its way to its northern-most centrifugal latitude at a $S_{I I I}$ longitude of $\sim 200^{\circ}$. Such a trend is similar to the one reported for the IFP for the same longitude range (Bonfond et al., 2013b). Because the southern Alfvén wing crosses a larger portion of the plasma sheet and yet the southern spot is more powerful, this indicates that the magnetic field asymmetry is the most likely source of the brightness asymmetry. In this $S_{I I I}$ longitude range, magnetic field models predict a stronger field intensity in the North than in the South.

The exposure time used to draw the plot is $30 \mathrm{~s}$, while the time step between two points is $10 \mathrm{~s}$. The short exposures made possible by the use of the timetag mode also reveal the large variability of the Europa footprint on timescales of minutes. Despite the weakness of the EFP, this $\sim 50 \%$ variability is not an artefact of a poor signal to noise ratio, as demonstrated by the error bars on Fig. 4. This plot shows a zoom on the last points of the previous plot, with the abscissa expressed in units of time rather than lon- 


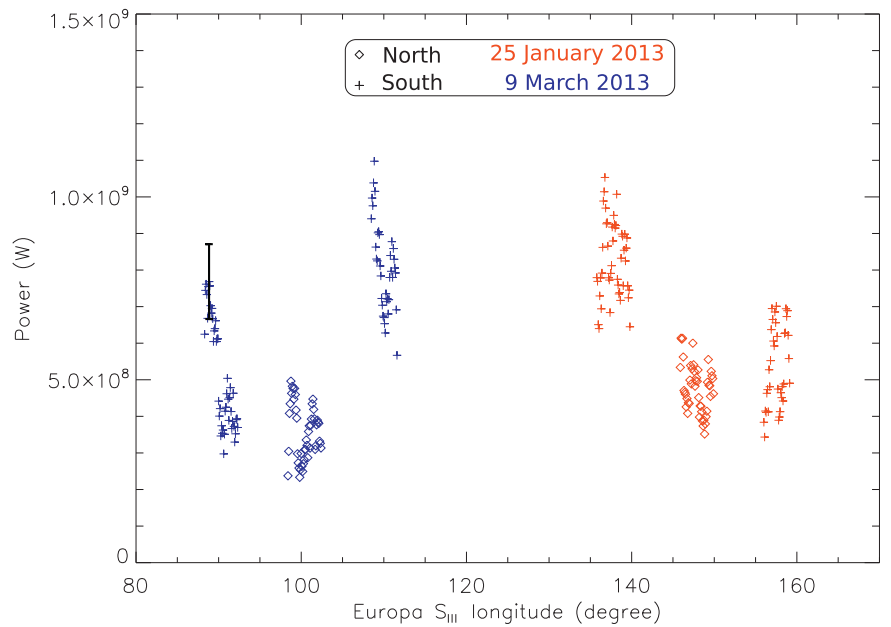

Fig. 3. Plot of the Europa footprint main spot emitted power. The stars and diamonds represent observations in the northern hemisphere and in the southern hemisphere, respectively. Each color accounts for a specific date. Assuming that the noise can be modelled as a Poisson process, the uncertainty estimate is computed as the square root of the total number of counts in the region of interest before background subtraction. An error bar representative for all cases is shown in black. (For interpretation of the references to color in this figure legend, the reader is referred to the web version of this article.)

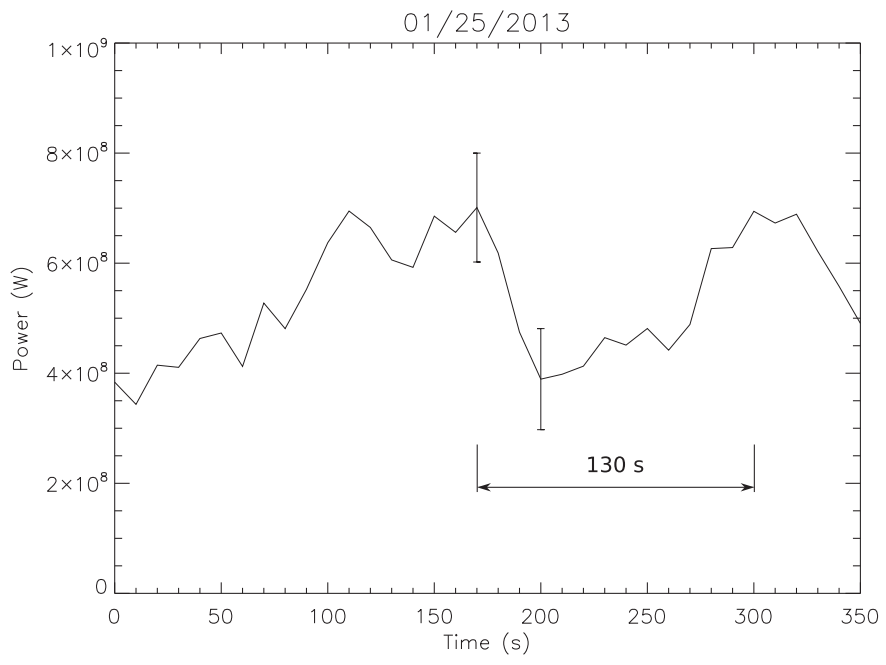

Fig. 4. Zoom on the last series of points of Fig. 3, except that the x-axis is expressed in units of time rather than longitudes. Significant brightness variations on timescales of $\sim 2$ min can be seen. The error bars are computed assuming that the counts in the region of interest follow a Poisson distribution.

gitudes. The time interval between two consecutive peaks is $130 \mathrm{~s}$. Such a short timescale variability had already been highlighted for the Io and the Ganymede footprints (Bonfond et al., 2007; Grodent et al., 2009). It has been attributed to either a signature of pulsed reconnection on the Ganymede magnetopause (Jia et al., 2008; Grodent et al., 2009) or to a signature of the quasi-periodic apparition and migration of double layer structures in the Jovian ionosphere (Hess et al., 2009; Bonfond et al., 2013b). While the first explanation can only be valid for the Ganymede footprint, the second one might apply for the three footprints. It is noteworthy that the two processes may also take place at the same time.

Fig. 5 is similar to Fig. 3, but for the Ganymede footprint. In some images of the southern hemisphere, a second (downstream) spot was also apparent and its power is shown with an $\mathrm{x}$ symbol while the main spot is shown with a + symbol. This downstream spot is systematically brighter than the upstream one, a situation that has occasionally been seen for the Io footprint in the southern

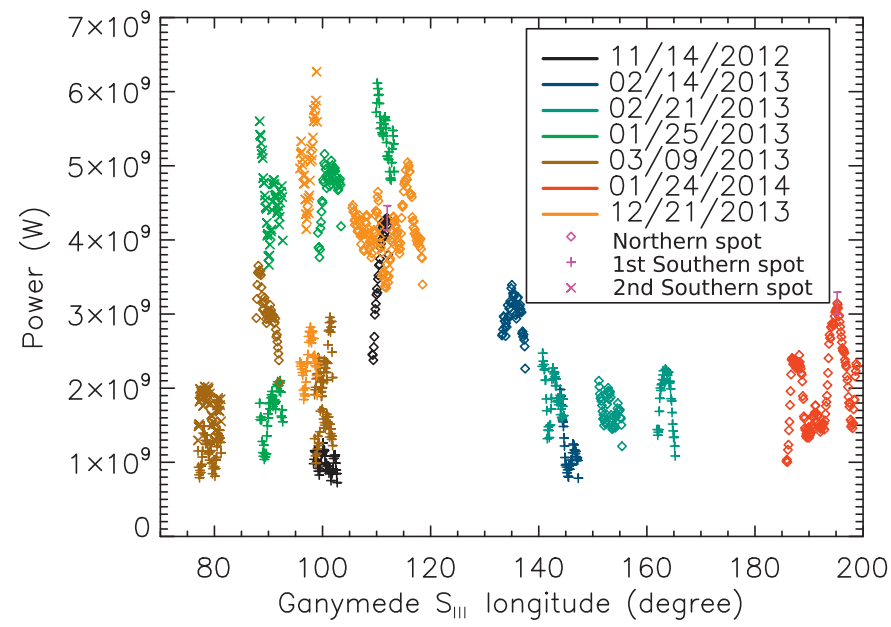

Fig. 5. Plot of the Ganymede footprint spots emitted power. The diamonds and crosses represent observations in the northern hemisphere and in the southern hemisphere, respectively. When two spots are visible in the southern hemisphere, the first one is shown with $\mathrm{a}+$ cross and the second one with a $\mathrm{x}$ cross. Each color accounts for a specific date. The error bar shown in pink is representative for all cases and is computed assuming that the counts in the region of interest follow a Poisson distribution. (For interpretation of the references to color in this figure legend, the reader is referred to the web version of this article.)

hemisphere for the same longitude range (Bonfond, 2010; Bonfond et al., 2013b). In the northern hemisphere, only one spot can be identified and its emitted power is reported with diamond symbols. Surprisingly and contrary to the two other footprints, there is no clear trend as to which hemisphere has the brightest spots. From one day to another, the absolute and relative power of the spots for an identical longitude range can be very different. For example, in the 100 to $115^{\circ}$ range, only one spot can be identified in each hemisphere and the northern spot is much brighter than the southern one on 14 November 2012, while the southern spot is slightly brighter on 25 January 2013.

The reason of such large discrepancies from one observation to another is unclear. First of all, in the southern images, the spots are very close to the limb and to the main emission. We tried to mitigate this contamination by carefully subtracting background emissions from the vicinity of the spots. However, because the background selection is manual and, more importantly, because short scale fluctuations of the background emission cannot be totally excluded, some hard-to-estimate (most probably $<20 \%$ ) uncertainty still remains and has not been accounted for to draw the error bars on Fig. 5. Assuming that these values are correct, one possible reason for the differences from one day to another would lie in the varying plasma condition (plasma density, temperature, composition and magnetic field strength) encountered by the satellite. For example, these parameters will affect the variability the power of the wave energy generated at Ganymede, but these parameters may also affect the filamentation of the Alfvén waves as well as the transmission coefficients at the plasma sheet boundary.

It is noteworthy that large (a factor of 2) and significant shorttimescale (2-3 min) brightness variations of the GFP are also reported in this dataset, in accordance to previous results (Grodent et al., 2009) and similarly the variability of the EFP reported here above.

\section{Length of the Europa footprint's spot}

The size of the footprint spots is expected to be related to the size of the interaction region of the satellite. It is however important to be clear about what is meant by this "size", as the footprint spots have a length along the footpath (broadly longitudinal), 
a width across this footpath (broadly latitudinal) and a vertical extent. For example, the IFP MAW spot is $\sim 850 \mathrm{~km}$ long (corresponding 3-4 Io diameters), $<200 \mathrm{~km}$ wide (corresponding to $<2$ Io diameters) (Bonfond, 2010). Indeed, the azimuthal extent of the Alfvén wing away from the satellite is larger than its radial extent, due to the pile-up of the field lines at the front of the obstacle. Moreover, additional effects due to the non-linear nature of the interaction can further increase the width of the Alfvén wing in the azimuthal direction (Jacobsen et al., 2007). The width of the auroral spots is thus expected to provide a more reliable estimate of the size of the interaction region at the moon, but it is much more difficult to measure on the HST images and not good case has been found for the EFP.

Grodent et al. (2006) reported a maximum value of $1100 \mathrm{~km}$ for the FWHM for the length of the Europa footprint's spot, corresponding to $\sim 15$ times the size of Europa mapped to the Jovian ionosphere along magnetic field lines. Such a value is larger, both relatively and in absolute value, than the Io footprint main spot ( $\sim 850 \mathrm{~km}$, corresponding 3-4 Io diameters (Bonfond, 2010)). This result is surprising, since it would indicate that the interaction region at Europa would be $\sim 4$ times larger than the interaction region at Io, despite the much thicker atmosphere and larger gas outflow rate of the latter. However, this result was obtained from one unique case, measured on a smoothed sum of 16100 s-long exposures acquired with the ACS instrument (0.033 arcsec/pixel, 3-pixel wide PSF). Here we re-examine the length of the Europa footprint main spot based on 5 timetag sequences and 17 ACCUM images acquired with the STIS instrument (0.024 arcsec/pixel, 1pixel wide PSF). In order to only keep images with the footprint as perpendicular as possible to the line of sight, we restricted our selection to spots located less than $30^{\circ}$ away from the CML (central meridian line). Moreover, the long time-tag sequences were split into several consecutive 100s sub-exposures. As a consequence, the total number of images in consideration is 40: 22 for the North and 18 for the South.

The method used to measure the length of the EFP is similar to the one used by Bonfond (2010) for the Io footprint MAW spot. It consists in building a stripe containing the EFP as shown on the top of Fig. 6. The X-direction corresponds to the mapping of the reference contour of the EFP on the image, with each pixel of the stripe corresponding to $25 \mathrm{~km}$ on the planet. The Y-direction corresponds to altitudes ranging from $525 \mathrm{~km}$ to $1275 \mathrm{~km}$ with steps of $75 \mathrm{~km}$. The brightness is then accumulated over $675 \mathrm{~km}$ centred on the brightness peak along the vertical direction in order to obtain a brightness profile. On the example shown on the top of Fig. 6, this corresponds to the space between the yellow lines. The background emission next to the EFP is removed (shown as a thick line) and the profile is further smoothed over $150 \mathrm{~km}$ in order to measure the FWHM, as illustrated in the plot at the bottom of Fig. 6.

In the northern (southern) hemisphere, the mean FWHM of the EFP spot main spot is $\sim 555 \mathrm{~km}(620 \mathrm{~km})$, the median value is $\sim 575 \mathrm{~km}(650 \mathrm{~km})$ and the standard deviation is $\sim 235 \mathrm{~km}$ (215 km). Whatever the hemisphere, such values correspond to $\sim 6$ times the projected diameter of Europa along the magnetic field lines. This ratio is only slightly larger then the one observed for Io, using a similar method. Similarly to the Io case, we can only conclude that the size of the interaction region is $<6$ times the size of Europa itself.

\section{Footprint spots multiplicity}

\subsection{Ganymede footprint spots multiplicity}

The evolution of the distance between the MAW spot and a secondary spot as a function of the moon's $S_{I I I}$ longitude varies with

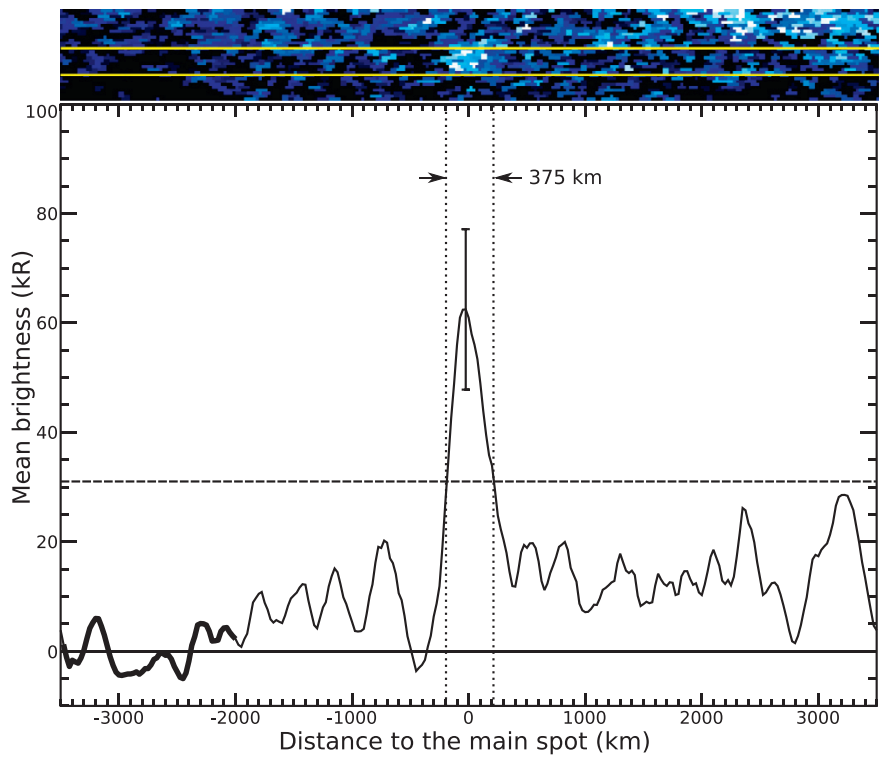

Fig. 6. Plot of the Europa footprint brightness profile based on the image acquired on 26 February 2003 at 00:49:15. The EFP stripe extracted from the image is shown on top and the plot is only based on the area between the yellow lines. The error bars are computed assuming that the counts in the region of interest follow a Poisson distribution. The horizontal dashed line is set at half the peak brightness and the two dotted vertical lines show the width at half maximum. The thicker portion of the profile indicates the area where the residual background is selected before subtraction. (For interpretation of the references to color in this figure legend, the reader is referred to the web version of this article.)

both the nature of the latter (i.e. either a TEB or a RAW spot) and the hemisphere in consideration. For a TEB spot, the distance is null when the moon is at the center of the plasma torus/sheet and maximum on the edge, whatever the hemisphere (but it will appear either upstream or downstream of the MAW spot). For a RAW spot in the northern hemisphere, the distance is maximum when it moon is at its northern-most centrifugal longitude (i.e. around $200^{\circ} \mathrm{S}_{I I I}$ ) and minimum when it moon is at its southernmost centrifugal longitude (i.e. around $20^{\circ} \mathrm{S}_{I I I}$ ), and vice-versa for the southern RAW spot (see the bottom panel of Fig. 8). Bonfond et al. (2013a) reported the identifications of pairs of spots belonging to the Ganymede footprint. They also showed that the relative motion between these spots is consistent with the motion between a MAW spot and a TEB spot. However, all the reported cases took place in the southern hemisphere. Images from 13 January 2014 distinctly show the presence of a pair of spots whose motion in polar maps fixed in System III relate to the Ganymede footprint (see the top panel of Fig. 7). At this time, Ganymede was located at $59^{\circ} \mathrm{S}_{I I I}$ at the beginning of the sequence and at $66^{\circ} \mathrm{S}_{I I I}$ at the end of the sequence. For these longitudes, Ganymede is located south-ward of the center of the plasma sheet and approaches it. The spots were initially $\sim 6000 \mathrm{~km}$ apart and this distance progressively diminishes to $\sim 2200 \mathrm{~km} 45$ min later (see the diamond symbols on Fig. 8). Qualitatively, the motion of these spots is fully consistent with the interpretation by Bonfond et al. (2013a) of this pair of spots in the southern hemisphere, i.e. with an upstream spot being a TEB spot and the generally brighter spot being a MAW spot. Should the two spots be a MAW spot and a RAW spot, the inter-spot distance would have increased, contrary to the observation. Finally, the rapid decrease of the inter-spot distance is possibly related to the presence of a magnetic anomaly in this longitude range, since the surface magnetic field is expected to be quite weak there (Grodent et al., 2008).

In light of this finding, the whole database of HST images acquired with the STIS and ACS instruments has been reinvestigated. 

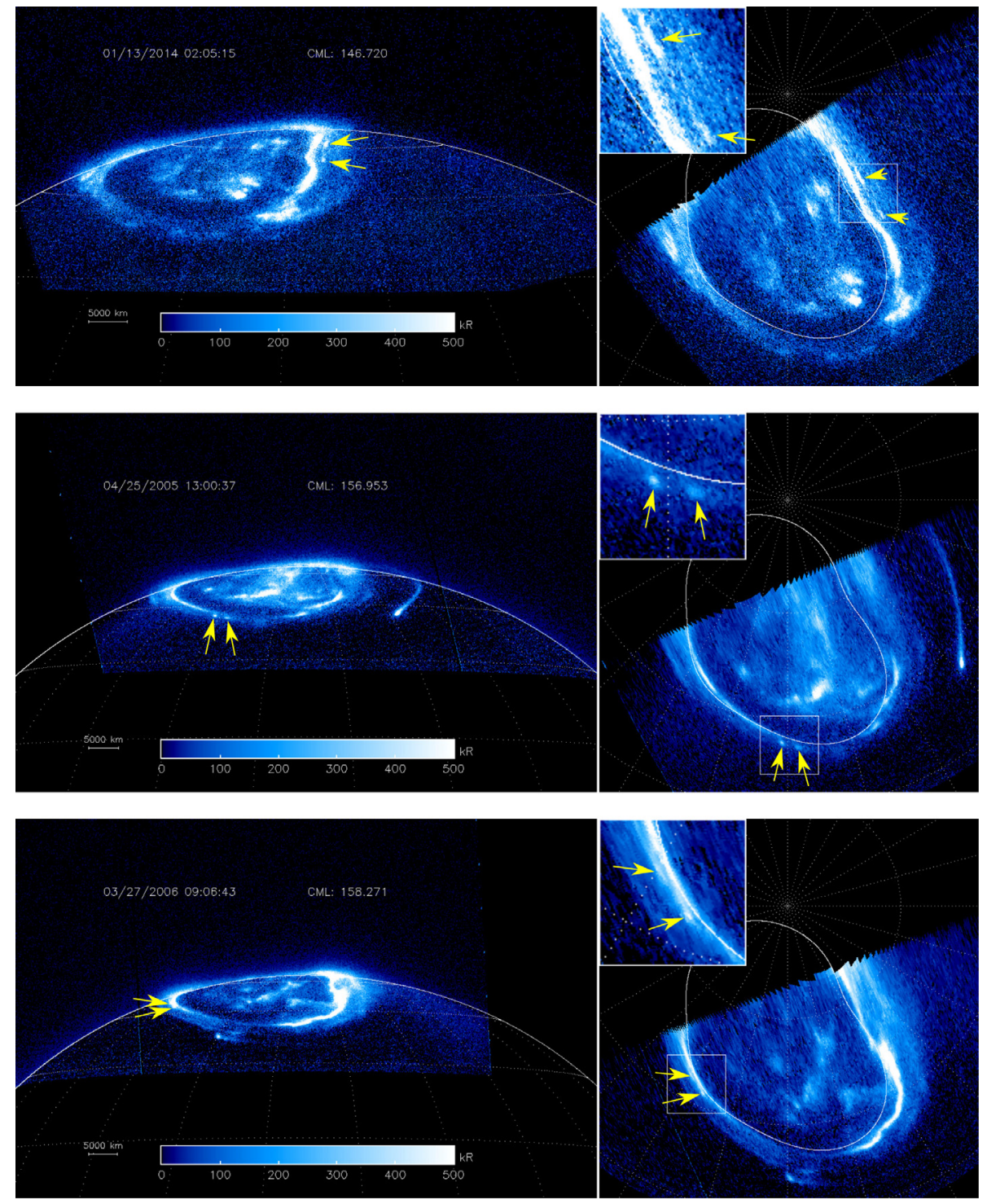

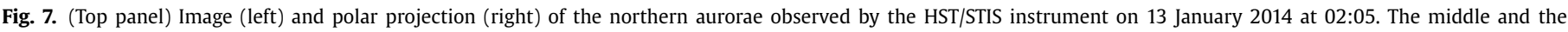

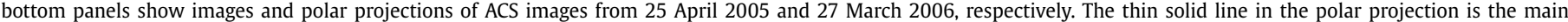
oval reference contour from February 2007 (i.e. compressed oval, Bonfond et al., 2012). The arrows highlight the pair of spots belonging to the Ganymede footprint.

No observation carried out in the same longitude range displays a pair of spots. However, two other clear cases of spot pairs are found on 25 April 2005 and on 27 March 2006 (also shown in Fig. 7). Ganymede was in the $S_{I I I}$ longitude range between $189^{\circ}$ and $212^{\circ}$ in the first case and between $258^{\circ}$ and $281^{\circ}$ in the second case. At these longitudes, Ganymede is at its northern-most centrifugal latitude (around $200^{\circ} S_{I I I}$ ) and comes back towards the center of the plasma sheet. Again, the spots appear to get closer to each other as time passes. However, in this longitude range, both scenarios (i.e. the secondary spot being either a TEB spot or a RAW spot) would lead to the same behavior.

\subsection{Europa footprint spots multiplicity}

As discussed in Section 3.1, the Europa footprint is generally the weakest of the three known footprints on Jupiter. Even when it is supposed to evolve in regions devoid of injection signatures or other auroral emissions, its brightness can be so low that it falls below the detection threshold ( $\mathrm{a}$ few $\mathrm{kR}$, depending on the instrument and filter in use, but mostly on the background auroral emis- sions). This weakness is related to the lower amount of Poynting flux generated at Europa compared to Io and Ganymede, which is related to the source, strength and size of the interaction region (Hess et al., 2011a; Saur et al., 2013).

Despite this difficulty, a careful inspection of all images for which geometry is compatible with the observation of the Europa footprint results in the discovery of a pair of spots belonging to this footprint in the southern hemisphere (Fig. 9). This HST orbit took place on 15 August 1999 and consists of three images acquired at 14:25, 14:30 and 14:41 in the southern hemisphere and one at 14:54 in the northern hemisphere. In $S_{\text {III }}$ fixed polar projection, one can clearly distinguish a pair of spots moving synchronously close to the expected location of the feet of the magnetic field lines passing through Europa in the last two images in the South. Such a motion is typical of footprints (the Io footprint moves in a similar fashion on the same sequence) and clearly differs from all the other auroral features of the outer emissions. An elongated spot is also present downstream of the main spot in the subsequent image in the North. On the first image of the sequence, a secondary spot cannot be clearly identified. The Eu- 


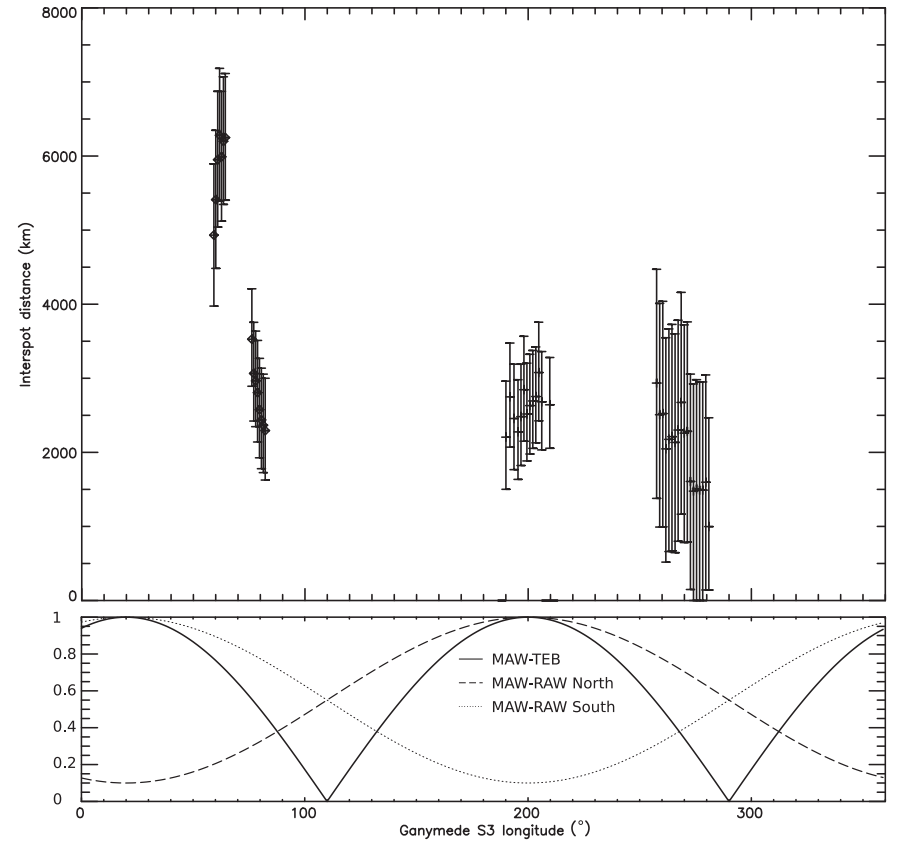

Fig. 8. (Top) Inter-spot distance between the two spots of the Ganymede footprint in the northern hemisphere. The diamonds represent STIS observations and the + signs represent ACS observations. The error bars assume a selection uncertainty of 1 pixel for the first spot and 2 pixels for the second one. (bottom) The solid line qualitatively shows the expected dependence of the distance for a transhemispheric electron beam spot. These distances are shown in arbitrary units because the nature of the secondary spot (TEB or RAW) and the exact Alfvén travel time is a priori unknown (and varies from footprint to footprint). The dashed and dotted lines represent the expected dependence of the distance for a reflected Alfvén wing spot in the northern and southern hemispheres, respectively. The decreasing distance in the $50-90^{\circ}$ strongly suggests that the observed secondary spot is a TEB spot.

ropa $S_{I I I}$ longitude is $136^{\circ}, 139^{\circ}, 145^{\circ}$ and $151^{\circ}$, respectively. For this longitude range, Europa is essentially halfway between the sheet's center $\left(\sim 110^{\circ} S_{I I I}\right)$ and its northern-most centrifugal latitude $\left(\sim 200^{\circ} S_{\text {III }}\right)$. The inter-spot distance is $1350 \mathrm{~km}$ and $1600 \mathrm{~km}$ for the two southern images and $3000 \mathrm{~km}$ for the northern one.

When the location of the spots are mapped back into the equatorial plane according to the EFP reference oval (Hess et al., 2011b), the inter-spot distance represents a longitudinal interval of $2.5^{\circ}, 3^{\circ}$ (South) and $12^{\circ}$ (North). A larger inter-spot distance in the North compared to the South while Europa is northward of the plasma sheet's center is compatible with these secondary spots being RAW spots, rather than TEB spots.

It should be noted, that in the case of the IFP, where both RAW and TEB spots are simultaneously observed, the relative brightness of these spots varies with System III (Bonfond et al., 2013b). Moreover, in Section 3.2, we have seen that not only the absolute brightness, but also the relative brightness of the GFP spots could vary from one observation to another for a given $S_{\text {III }}$ longitude. Should the EFP behave in the same way, then it might explain the rarity (one unique case so far) of the observation of a secondary spot as bright as the main one.

\section{Conclusions}

The complexity and the variability Io footprint morphology can be successfully explained by a combination of Alfvén waves partial reflection and the generation of electron beams in one hemisphere precipitating into the opposite hemisphere (Bonfond et al., 2008; 2013b; Hess et al., 2010; 2013a). However if this scenario is correct, then it was not clear why the other footprints did not show the same features and variability as the Io footprint. In the present study, based on a new and better suited dataset of HST STIS timetag sequences, completed by the wealth of high resolution images gathered by the STIS and ACS cameras since 1997, we show that the Europa and Ganymede footprints actually display, at least occasionally, the same morphology and behavior as the Io footprint. Specifically, we show here examples where the Europa footprint and the Ganymede footprint can both be made of at least two spots. Moreover, we show that the fluctuations of the inter-spot distances and the spots' brightness of the EFP and GFP are also formally similar to those observed for the Io footprint. It is noteworthy that these observations constitutes an argument in favor of the universality of the auroral outcome of the satellitemagnetosphere interactions.

In the case of the Ganymede footprint, we now have multiple observations of pairs of spots in both hemispheres and the inter-spot distances are compatible with the scenario of transhemispheric electron beams. However, the secondary spots are not systematically observed on the HST images, perhaps due to the limited sensitivity of the detectors. It is also shown that both the absolute and relative brightness of the spots, as well as the interspot distance (Bonfond et al., 2013a), strongly varies from one observation to another. This suggests that the state of the plasma environment (i.e. the plasma density, temperature and composition, together with the magnetic field strength) interacting with Ganymede most probably controls these parameters. Additionally, a sequence during which the GFP main spot vanishes for several minutes as it crosses an injection signature has been identified, further strengthening this conclusion. It is noteworthy that a similar case of disappearance of the IFP while crossing an injection signature has already been reported (Bonfond et al., 2012).

In the case of the Europa footprint, the only detection of secondary spots relies on observations carried on a unique day. Fortunately, this set of images is made of quasi-simultaneous images of both hemispheres and hints of a secondary spot are seen both in the South and in the North. A larger inter-spot distance in the northern hemisphere than in the southern one while Europa is north-ward of the plasma sheet suggests that the downstream spot is a Reflected Alfvén Wing spot. This implies that the density gradients in the plasma sheet can (at least occasionally) be large enough to trigger significant reflections of the Alfvén waves launched at Europa.

While at Io, both types of secondary spots are seen (with variable relative and absolute brightnesses as a function of System III), it remains surprising that one type dominates at Europa and another one at Ganymede. It should nevertheless be noted that the number of positive detections remains small. Additionally and similarly to the IFP case (Bonfond et al., 2013b), the relative and absolute brightness of the spots can significantly vary from one observation to another. Furthermore, secondary spots are not always seen, even for observations carried out in similar geometries. A detailed theoretical analysis of all the processes leading to the various spots of the satellite footprints would thus be required to explain this apparent discrepancy. On the other hand, more detailed observations from Juno's UV Spectrograph (UVS) (Gladstone et al., 2014) might also help identifying spots too faint for HST's instruments. Similarly, close-up views of Saturn's aurorae may reveal additional spots for the Enceladus footprint. However, because Saturn's magnetic dipole axis and rotation axis are almost co-aligned, Enceladus does not move up and down the plasma sheet. Thus the relative motion of the spots will not help determining their nature.

A re-analysis of the size of the Europa footprint main spot, carried out on a larger and better suited dataset of STIS images, shows that FWHM of the footprint is $\sim 600 \mathrm{~km}$ and maps to area $\sim 6$ times larger than Europa in the azimuthal direction. Again, such a ratio is similar to the ratio found for the Io footprint, suggesting 

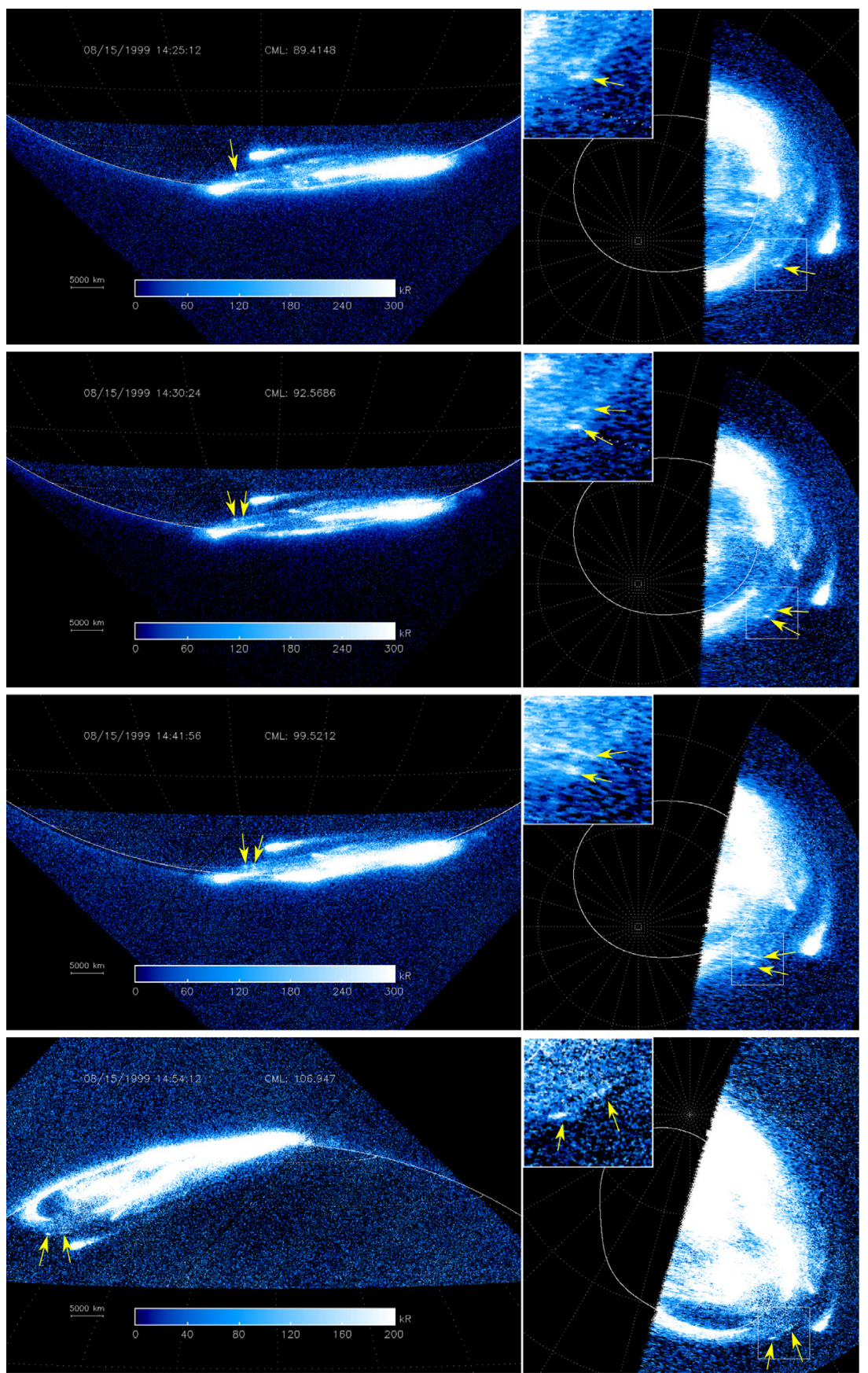

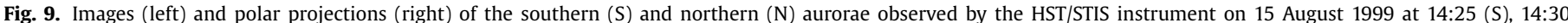

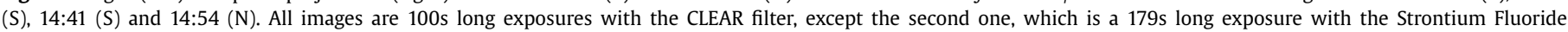

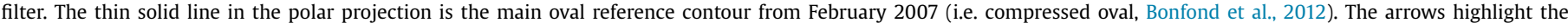
spots belonging to the Europa footprint. In the top images, only one spot appears to be part of the EFP, while two spots can be identified on the 3 subsequent ones.

that the processes at play are the same and that the interaction region is most likely limited to the satellite's extended atmosphere.

Finally, large brightness variations of the EFP's main spot have been identified on timescales of minutes, similarly to Io's and Ganymede's cases. A possible scenario for such a behavior that would work for all footprints could be related to the quasi-periodic formation of upward migrating double layer structures in the ionosphere (Hess et al., 2009; Bonfond et al., 2013b).

It is now clear that the plasma environment (particle energy distribution, density, composition, magnetic field strength, etc.) has a measurable influence on the footprints' spots relative and abso- lute brightness and position. Such obvious variabilities carries the hope that observations of the satellite footprints could provide a precious diagnostic of the state of the magnetosphere when and where in-situ measurements are not available. However, such a task would require quantitative estimates of all the processes at play and additional observational and modelling efforts will be necessary. Hence ongoing (HST, Cassini, Hisaki, Juno) and future (JUICE, Europa Mission) missions will provide unique opportunities to verify and calibrate the influence of the plasma environment on the footprints. 


\section{Acknowledgements}

B.B., D.G., A. R., J.-C.G. are supported by the PRODEX program managed by ESA in collaboration with the Belgian Federal Science Policy Office. B.B. was funded by the Fund for Scientific Research (F.R.S-FNRS). S.V.B. was supported by an STFC Ernest Rutherford Fellowship (ST/M005534/1). This research is based on observations with the NASA/ESA Hubble Space Telescope, obtained at the Space Telescope Science Institute, which is operated by AURA for NASA. It is based on publicly available observations acquired with the NASA/ESA Hubble Space Telescope (program IDs 7308, 7769, $8171,8657,10140,10507,10862,12883,13035$ ) and obtained from the Space Telescope Science Institute (https://archive.stsci.edu/hst/ search.php).

\section{References}

Badman, S.V., Bonfond, B., Fujimoto, M., Gray, R.L., Kasaba, Y., Kasahara, S., Kimura, T., Melin, H., Nichols, J.D., Steffl, A.J., Tao, C., Tsuchiya, F., Yamazaki, A., Yoneda, M., Yoshikawa, I., Yoshioka, K., 2016. Weakening of Jupiter's main auroral emission during january 2014. Geophys. Res. Lett. 43, 988-997. doi:10.1002/ 2015GL067366.

Bonfond, B., 2009. Morphology and Dynamics of the Io UV Footprint. Université de Liège Phd Thesis.

Bonfond, B., 2010. The 3-D extent of the Io UV footprint on Jupiter. J. Geophys. Res. 115 (A14), A09217. doi:10.1029/2010JA015475

Bonfond, B., Gérard, J.-C., Grodent, D., Saur, J., 2007. Ultraviolet Io footprint short timescale dynamics. Geophys. Res. Lett. 34, 6201. doi:10.1029/2006GL028765.

Bonfond, B., Grodent, D., Gérard, J.-C., Radioti, A., Saur, J., Jacobsen, S., 2008. UV Io footprint leading spot: a key feature for understanding the UV lo footprint multiplicity? Geophys. Res. Lett. 35, 5107. doi:10.1029/2007GL032418.

Bonfond, B., Grodent, D., Gérard, J.-C., Stallard, T., Clarke, J.T., Yoneda, M., Radioti, A., Gustin, J., 2012. Auroral evidence of Io's control over the magnetosphere of Jupiter. Geophys. Res. Lett. 39, 1105. doi:10.1029/2011GL050253.

Bonfond, B., Hess, S., Bagenal, F., Gérard, J.-C., Grodent, D., Radioti, A., Gustin, J., Clarke, J.T., 2013a. The multiple spots of the Ganymede auroral footprint. Geophys. Res. Lett. 40, 4977-4981. doi:10.1002/grl.50989.

Bonfond, B., Hess, S., Gérard, J.-C., Grodent, D., Radioti, A., Chantry, V., Saur, J., Jacobsen, S., Clarke, J.T., 2013b. Evolution of the Io footprint brightness I: Far-UV observations. Planet. Space Sci. 88, 64-75. doi:10.1016/j.pss.2013.05.023.

Bonfond, B., Vogt, M.F., Gérard, J.-C., Grodent, D., Radioti, A., Coumans, V., 2011. Quasi-periodic polar flares at Jupiter: a signature of pulsed dayside reconnections? Geophys. Res. Lett. 380, L02104. doi:10.1029/2010GL045981.

Chust, T., Roux, A., Kurth, W.S., Gurnett, D.A., Kivelson, M.G., Khurana, K.K., 2005. Are Io's Alfvén wings filamented? Galileo observations. Planet. Space Sci. 53, 395-412. doi:10.1016/j.pss.2004.09.021.

Clarke, J.T., Ajello, J., Ballester, G., Ben Jaffel, L., Connerney, J., Gérard, J.-C., Gladstone, G.R., Grodent, D., Pryor, W., Trauger, J., Waite, J.H., 2002. Ultraviolet emissions from the magnetic footprints of Io, Ganymede and Europa on Jupiter. Nature 415, 997-1000.

Clarke, J.T., Ballester, G.E., Trauger, J., Evans, R., Connerney, J.E.P., Stapelfeldt, K. Crisp, D., Feldman, P.D., Burrows, C.J., Casertano, S., Gallagher III, J.S., Griffiths, R.E., Hester, J.J., Hoessel, J.G., Holtzman, J.A., Krist, J.E., Meadows, V., Mould, J.R., Scowen, P.A., Watson, A.M., Westphal, J.A., 1996. Far-ultraviolet imaging of Jupiter's aurora and the lo "Footprint". Science 274, 404-409.

Connerney, J.E.P., Baron, R., Satoh, T., Owen, T., 1993. Images of excited $H_{3}^{+}$at the foot of the Io flux tube in Jupiter's atmosphere. Science 262, 1035-1038.

Connerney, J.E.P., Satoh, T., 2000. The $H_{3}^{+}$ion: a remote diagnostic of the jovian magneto sphere. In: Astronomy, physics and chemistry of $\mathrm{H}+3$, pp. 2471-+

Dumont, M., Grodent, D., Radioti, A., Bonfond, B., Gérard, J.-C., 2014. Jupiter's equatorward auroral features: possible signatures of magnetospheric injections. J. Geophys. Res. 119, 10068. doi:10.1002/2014JA020527.

Gérard, J.-C., Bonfond, B., Grodent, D., Radioti, A., Clarke, J.T., Gladstone, G.R., Waite, J.H., Bisikalo, D., Shematovich, V.I., 2014. Mapping the electron energy in Jupiter's aurora: hubble spectral observations. J. Geophys. Res. doi:10.1002/ 2014JA020514. http://dx.doi.org/10.1002/2014JA020514

Gérard, J.-C., Saglam, A., Grodent, D., Clarke, J.T., 2006. Morphology of the ultraviolet Io footprint emission and its control by Io's location. J. Geophys. Res. 111, 4202+. doi:10.1029/2005JA011327.

Gladstone, G.R., Persyn, S.C., Eterno, J.S., Walther, B.C., Slater, D.C., Davis, M.W., Versteeg, M.H., Persson, K.B., Young, M.K., Dirks, G.J., Sawka, A.O., Tumlinson, J., Sykes, H., Beshears, J., Rhoad, C.L., Cravens, J.P., Winters, G.S., Klar, R.A., Lockhart, W., Piepgrass, B.M., Greathouse, T.K., Trantham, B.J., Wilcox, P.M., Jackson, M.W., Siegmund, O.H.W., Vallerga, J.V., Raffanti, R., Martin, A., Gérard, J.-C., Grodent, D.C., Bonfond, B., Marquet, B., Denis, F., 2014. The ultraviolet spectrograph on NASA's Juno mission. Space Sci. Rev. doi:10.1007/s11214-014-0040-z.

Goldreich, P., Lynden-Bell, D., 1969. Io, a jovian unipolar inductor. Astrophys. J. 156, 59-78.
Grodent, D., Bonfond, B., Gérard, J.-C., Radioti, A., Gustin, J., Clarke, J.T., Nichols, J., Connerney, J.E.P., 2008. Auroral evidence of a localized magnetic anomaly in Jupiter's northern hemisphere. J. Geophys. Res. 113 (A12), 9201-+. doi:10.1029/ 2008JA013185

Grodent, D., Bonfond, B., Radioti, A., Gérard, J.-C., Jia, X., Nichols, J.D., Clarke, J.T., 2009. Auroral footprint of Ganymede. J. Geophys. Res. 114, 7212-+. doi:10.1029/ 2009JA014289.

Grodent, D., Gérard, J.-C., Gustin, J., Mauk, B.H., Connerney, J.E.P., Clarke, J.T., 2006. Europa's FUV auroral tail on Jupiter. Geophys. Res. Lett. 33, 6201-+. doi:10.1029/ 2005 GL025487.

Gustin, J., Bonfond, B., Grodent, D., Gérard, J.C., 2012. Conversion from HST ACS and STIS auroral counts into brightness, precipitated power and radiated power for h2 giant planets j.. J. Geophys. Res. 117 (A16), 7316. doi:10.1029/2012JA017607.

Gustin, J., Grodent, D., Ray, L.C., Bonfond, B., Bunce, E.J., Nichols, J.D., Ozak, N., 2016 Characteristics of north jovian aurora from STIS FUV spectral images. Icarus 268, 215-241. doi:10.1016/j.icarus.2015.12.048.

Hess, S., Bonfond, B., Chantry, V., Gérard, J.-C., Grodent, D., Jacobsen, S., Radioti, A. 2013. Evolution of the Io footprint brightness II: modeling. Planet. Space Sci..

Hess, S., Zarka, P., Mottez, F., Ryabov, V.B., 2009. Electric potential jumps in the IoJupiter flux tube. Planet. Space Sci. 57, 23-33. doi:10.1016/j.pss.2008.10.006

Hess, S.G.P., Delamere, P.A., Dols, V., Ray, L.C., 2011a. Comparative study of the power transferred from satellite-magnetosphere interactions to auroral emissions. J. Geophys. Res. 116, A01202. doi:10.1029/2010JA015807.

Hess, S.L.G., Bonfond, B., Delamere, P.A., 2013b. How could the Io footprint disappear? Planet. Space Sci. 89, 102-110. doi:10.1016/j.pss.2013.08.014.

Hess, S.L.G., Bonfond, B., Zarka, P., Grodent, D., 2011b. Model of the Jovian magnetic field topology constrained by the Io auroral emissions. J. Geophys. Res. 116, A05217. doi:10.1029/2010JA016262.

Hess, S.L.G., Delamere, P., Dols, V., Bonfond, B., Swift, D., 2010. Power transmission and particle acceleration along the Io flux tube. J. Geophys. Res. 115, A06205. doi:10.1029/2009JA014928.

Jacobsen, S., Neubauer, F.M., Saur, J., Schilling, N., 2007. Io's nonlinear MHD-wave field in the heterogeneous Jovian magnetosphere. Geophys. Res. Lett. 34, 10202+. doi:10.1029/2006GL029187.

Jacobsen, S., Saur, J., Neubauer, F.M., Bonfond, B., Gérard, J.-C., Grodent, D., 2010. Location and spatial shape of electron beams in Io's wake. J. Geophys. Res. 115, A04205. doi:10.1029/2009JA014753.

Jia, X., Kivelson, M.G., Khurana, K.K., Walker, R.J., 2009. Magnetic fields of the satellites of Jupiter and Saturn. Space Sci. Rev. 152, 271-305. doi:10.1007/ s11214-009-9507-8.

Jia, X., Walker, R.J., Kivelson, M.G., Khurana, K.K., Linker, J.A., 2008. Threedimensional MHD simulations of Ganymede's magnetosphere. J. Geophys. Res. 113, 6212-+. doi:10.1029/2007JA012748.

Jones, S.T., Su, Y.-J., 2008. Role of dispersive Alfvén waves in generating parallel electric fields along the Io-Jupiter fluxtube. J. Geophys. Res. 113, 12205-+. doi:10.1029/2008JA013512.

Mauk, B.H., Clarke, J.T., Grodent, D., Waite, J.H., Paranicas, C.P., Williams, D.J., 2002. Transient aurora on Jupiter from injections of magnetospheric electrons. Nature 415, 1003-1005.

Neubauer, F.M., 1980. Nonlinear standing Alfven wave current system at Io - Theory. J. Geophys. Res. 85, 1171-1178.

Payan, A.P., Rajendar, A., Paty, C.S., Crary, F., 2014. Effect of plasma torus density variations on the morphology and brightness of the Io footprint. J. Geophys. Res. 119, 3641-3649. doi:10.1002/2013JA019299.

Prangé, R., Rego, D., Southwood, D., Zarka, P., Miller, S., Ip, W., 1996. Rapid energy dissipation and variability of the Io-Jupiter electrodynamic circuit. Nature 379, 323-325. doi:10.1038/379323a0.

Pryor, W.R., Rymer, A.M., Mitchell, D.G., Hill, T.W., Young, D.T., Saur, J., Jones, G.H., Jacobsen, S., Cowley, S.W.H., Mauk, B.H., Coates, A.J., Gustin, J., Grodent, D., Gérard, J.-C., Lamy, L., Nichols, J.D., Krimigis, S.M., Esposito, L.W., Dougherty, M.K., Jouchoux, A.J., Stewart, A.I.F., McClintock, W.E., Holsclaw, G.M., Ajello, J.M., Colwell, J.E., Hendrix, A.R., Crary, F.J., Clarke, J.T., Zhou, X., 2011 The auroral footprint of Enceladus on Saturn. Nature 472, 331-333. doi:10.1038/ nature09928.

Saur, J., Grambusch, T., Duling, S., Neubauer, F.M., Simon, S., 2013. Magnetic energy fluxes in sub-Alfvénic planet star and moon planet interactions. Astron. Astrophys. 552, A119. doi:10.1051/0004-6361/201118179.

Saur, J., Neubauer, F.M., Connerney, J.E.P., Zarka, P., Kivelson, M.G., 2004. Jupiter. The Planet, Satellites and Magnetosphere. Cambridge University Press. Plasma interaction of Io with its plasma torus pp. 537--560

Tao, C., Kimura, T., Badman, S.V., Murakami, G., Yoshioka, K., Tsuchiya, F., André, N., Yoshikawa, I., Yamazaki, A., Shiota, D., Tadokoro, H., Fujimoto, M., 2016. Variation of Jupiter's aurora observed by Hisaki/EXCEED: 1. Observed characteristics of the auroral electron energies compared with observations performed using HST/STIS. J. Geophys. Res. 121, 4041-4054. doi:10.1002/2015JA021271.

Vasavada, A.R., Bouchez, A.H., Ingersoll, A.P., Little, B., Anger, C.D., Team, T.G.S., 1999. Jupiter's visible aurora and Io footprint. J. Geophys. Res. 104, 27133-+

Wannawichian, S., Clarke, J.T., Nichols, J.D., 2010. Ten years of Hubble Space Telescope observations of the variation of the Jovian satellites' auroral footprint brightness. J. Geophys. Res 115, A02206. doi:10.1029/2009JA014456.

Williams, D.J., Mauk, B.H., McEntire, R.E., Roelof, E.C., Armstrong, T.P., Wilken, B., Roederer, J.G., Krimigis, S.M., Fritz, T.A., Lanzerotti, L.J., 1996. Electron beams and lon composition measured at lo and in its Torus. Science 274, 401-403. 